\title{
Challenges in the Production of Convalescent Hyperimmune Plasma in the Age of COVID-19
}

\author{
Massimo Franchini, MD ${ }^{1}$ Claudia Del Fante, MD ${ }^{2}$ Catherine Klersy, MD ${ }^{3}$ Claudia Glingani, BSc ${ }^{1}$ \\ Elena Percivalle, $\mathrm{BSc}^{4}$ Fausto Baldanti, MD, $\mathrm{PhD}^{4,5}$ Cesare Perotti, $\mathrm{MD}^{2}$
}

${ }^{1}$ Department of Hematology and Transfusion Medicine, Carlo Poma Hospital, Mantua, Italy

2 Immunohaematology and Transfusion Service, Apheresis and Cell Therapy Unit, Fondazione IRCCS Policlinico San Matteo, Pavia, Italy

${ }^{3}$ Biometry and Clinical Epidemiology Service, Fondazione IRCCS

Policlinico San Matteo, Pavia, Italy

${ }^{4}$ Molecular Virology Unit, Fondazione IRCCS Policlinico San Matteo, Pavia, Italy

${ }^{5}$ Department of Clinical, Surgical, Diagnostic and Pediatric Sciences, University of Pavia, Pavia, Italy

Semin Thromb Hemost 2020;46:804-806.

In December 2019, the first patients were diagnosed with coronavirus disease 2019 (COVID-19), a disease caused by severe acute respiratory syndrome coronavirus-2 (SARS-CoV$2)^{1}$ In only a few months, COVID-19 has spread globally, affecting over 3 million individuals with over 250,000 deaths (data updated on May 2). ${ }^{2}$ This has prompted the World Health Organization (WHO) to declare the COVID-19 outbreak a pandemic. $^{3}$ Unfortunately, no uniformly standardized therapy has been endorsed for COVID-19 and a number drugs are currently being investigated in several nonrandomized or randomized trials for patients with life-threatening COVID-19.,4 These agents includes steroids, chloroquine, antiviral medications (i.e., remdesivir, loparinir/ritonavir), and anti-inflammatory agents (i.e., tocilizumab, sarilumab, baricitinib), the latter being utilized following the discovery of the involvement of SARS$\mathrm{CoV}$-2-induced inflammatory response in the pathogenesis of SARS. 5,6

Experiences from previous coronavirus epidemics (SARS and Middle East Respiratory Syndrome) indicate that convalescent plasma collected from recovered COVID-19 patients, containing neutralizing antibodies against SARS-CoV-2, could be a potentially effective therapeutic weapon. ${ }^{7-13}$ In a pilot study of 10 patients with severe COVID- $19,{ }^{14}$ classified according to WHO Interim Guidance, ${ }^{15}$ the investigators collected convalescent plasma with neutralizing antibody titers $\geq 1: 640$ dilution. All 10 patients had a rapid improvement in symptoms and pulmonary lesions (within 1-3 days). ${ }^{14} \mathrm{~A}$ case series by Shen and colleagues on five critically ill patients with COVID19 further confirmed the potential benefit of this biological antiviral therapy. ${ }^{16}$ With this background, ${ }^{17}$ considering the
Address for correspondence Massimo Franchini, MD, Department of Hematology and Transfusion Medicine, Carlo Poma Hospital, Mantova, Italy (e-mail: massimo.franchini@asst-mantova.it).

dramatic situation and the high lethality rate of COVID-19 in Italy, we have planned a multicenter interventional single-arm trial (Clinicaltrials.gov:NCT04321421) to produce hyperimmune plasma for treating critical patients with COVID-19. ${ }^{18}$ Briefly, 500 to $600 \mathrm{~mL}$ plasmaphereses are collected from adult male donors, recovered from COVID-19 (defined as two consecutive nasopharyngeal swabs negative for reverse transcriptase-polymerase chain reaction [RT-PCR] testing), aged 18 years or older, through a cell separator device and evaluated for transmissible diseases (human immunodeficiency virus, hepatitis $C$ virus, hepatitis B virus, and syphilis) according to Italian laws. Additional molecular tests for detection of hepatitis A virus, hepatitis E virus, and parvovirus B19, and neutralizing titer for SARS-CoV-2 will be performed according to the recommendations from Italian National Blood Center (CNS) for donation of anti-COVID-19 convalescent plasma. ${ }^{19}$ Pathogen reduction of plasma is also performed before freezing or transfusion. Every plasmapheresis is divided into two 250 to $300 \mathrm{~mL}$ units, each of which is used to treat one patient with severe COVID-19 infection. A second infusion is also contemplated after 48 hours, in the case of lack of response to the first unit. The primary endpoint of the study is reduction of mortality rate. Other clinical endpoints, viral load (quantitative RT-PCR expressed as number of copies $/ \mathrm{mL}$ ), and immune response (expressed as neutralizing titer, which is the maximum dilution with a reduction of $90 \%$ of the cytopathic effect; a positive titer is equal to or greater than 1:10) are also considered. The sample size calculation involves the enrolment of 43 patients. ${ }^{18}$ Patients are continuously monitored for adverse events; these will be communicated to the local published online June 8, 2020
Issue Theme Maintaining Hemostasis and Preventing Thrombosis in COVID-19 -Part I; Guest Editors: Emmanuel J. Favaloro, PhD, FFSc (RCPA), and Giuseppi Lippi, MD.
Copyright $\odot 2020$ by Thieme Medical Publishers, Inc., 333 Seventh Avenue, New York, NY 10001, USA.

Tel: +1(212) 760-0888.
DOI https://doi.org/ 10.1055/s-0040-1713433. ISSN 0094-6176. 
Transfusion Service staff, who in turn will notify them to the national electronic system for hemovigilance (SISTRA; https:// www.centronazionalesangue.it). In addition, according to directives from $\mathrm{CNS}^{20}$ donors are advised to inform their Transfusion Service in the case of reappearance of symptoms compatible with SARS-CoV-2 infection within 14 days after donation (postdonation information).

The study started on March 17, 2020. During the conduct of the study, several challenges have emerged linked to the enrolment of convalescent donors. The first issue was with regards to the selection of convalescent plasma donors among patients recovered from COVID-19. Patient-donors aged over 70 years are usually excluded from plasma donation, according to Italian laws for blood donor selection, due to a hypothetically increased risk of apheresis-related adverse events. Similarly, pluriparous women are generally excluded from donation, because of increased risk of transfusion-related acute lung injury in patients receiving their plasma that contains high levels of antibodies against recipients' class I and II human leukocyte antigens. In addition, candidates for hyperimmune plasma donation must be in good health without current or pre-existing diseases, according to Italian laws on selection of voluntary unpaid blood donors. ${ }^{18}$ Information about the course of COVID-19 disease for the patient (e.g., severity, treatment, dates of onset and resolution of clinical symptoms) is usually requested prior to plasma collection. In our experience, approximately 30 to $40 \%$ of recovered patients initially screened are finally eligible for convalescent plasma donation, with comorbidities (i.e., cardiovascular, neoplastic, or autoimmune disorders), age ( $>70$ years) and sex (pluriparous females) the main causes for exclusion from plasma donation. In addition, CNS recommends that donors should be completely recovered by at least 14 days without a positive anamnesis for contacts with subjects with documented SARS-CoV-2 infection during this period. This could generate a problem for recovered health care personnel who thus must donate plasma before their return to operational medical COVID units. ${ }^{19,20} \mathrm{~A}$ consistent proportion of convalescent plasma donors is represented by recovered periodic voluntary blood donors belonging to blood donor associations and this is very important as it increases the safety of collected blood. Potential convalescent plasma donors are retrieved from a local registry of patients who were infected with COVID-19 and are usually contacted by telephone by a doctor or health care personnel who performs an initial anamnestic screening to discover possible contraindications to plasma donations. In our experience, due to the high number of phone calls required to provide adequate daily plasma apheresis donations (8-10/day), a health care professional must be dedicated exclusively to this activity.

Another critical point is the titer of anti-SARS-CoV-2 neutralizing antibodies. In our protocol, a minimum titer of 1:160 is required to collect plasma. Several serologic tests based on enzyme-linked immunosorbent assay (ELISA) technology for detection of specific immunoglobulin A ( $\operatorname{Ig} A), \operatorname{IgG}$, and $\operatorname{IgM}$ anti-SARS-CoV-2 antibodies are available and several studies have tried to correlate their titers with patients' disease severity, clinical outcomes, and with persistent immune protection of recovered patients against SARS-CoV-2. ${ }^{21-25}$ However, although these assays might be useful for preliminary screening of seropositive donors, the neutralization test (see Duan et $\mathrm{al}^{14}$ for a detailed description of the method) remains the gold standard for identification and quantification of antiSARS-CoV-2 neutralizing antibodies. This test, based on measurement of reduction of viral cytopathic effect in cell culture following addition of recovered patient's serum, does not necessarily correlate with anti-COVID-19 antibody levels measured by ELISA assays, but accurately quantifies the antiCOVID-19 neutralizing properties of donors' plasma, thereby giving a reliable indication of the in vivo antiviral potency of the convalescent plasma. Although very specific, this test is very technically demanding and requires 72 hours of incubation before results are available. The neutralization test is therefore very useful for clinical and experimental studies, but not for epidemiological investigations of seroprevalence. Our very preliminary experience suggests that anti-COVID-19 neutralizing antibodies reach their peak early, approximately 10 to 20 days from the disappearance of symptoms. Since we do not have clear data on the persistence of neutralizing levels in plasma over time, we recommend collecting convalescent plasma just after the 14-day period required by CNS for convalescent plasma donation, providing that two consecutive nasopharyngeal swabs are negative for COVID-19. Neutralization tests are usually performed on donors before convalescent plasma collection, to select those donors with titers $\geq 1: 160$ and to eliminate as few donations as possible.

In our opinion, beside the challenges previously mentioned, which will be mitigated by implementing our expertise in managing convalescent donors, the collection of plasma represents an attractive therapeutic option, also considering the possibility of collecting plasma by plasmapheresis procedures quickly and effectively. Furthermore, the possibility of having local donors offers the added value of providing specific immunity acquired against the infectious agent of the local strain, considering that in other areas the strain may be different. The final results of our trial will help to clarify the therapeutic role of anti-COVID-19 convalescent plasma.

Conflict of Interest

None declared.

\section{References}

1 Zhu N, Zhang D, Wang W, et al; China Novel Coronavirus Investigating and Research Team. A novel coronavirus from patients with pneumonia in China, 2019. N Engl J Med 2020; 382(08):727-733

2 WHO. Coronavirus disease (COVID-19) outbreak, 2020. Available at: https://www.who.int/emergencies/diseases/novel-coronavirus-2019. Accessed April 27, 2020

3 Zhang L, Liu Y. Potential interventions for novel coronavirus in China: a systematic review. J Med Virol 2020;92(05):479-490

4 Zhai P, Ding Y, Wu X, Long J, Zhong Y, Li Y. The epidemiology, diagnosis and treatment of COVID-19. Int J Antimicrob Agents 2020;55(05):105955

5 Zhou M, Zhang X, Qu J. Coronavirus disease 2019 (COVID-19): a clinical update. Front Med 2020;14(02):126-135 
6 Xie M, Chen Q. Insight into 2019 novel coronavirus - an updated interim review and lessons from SARS-CoV and MERS-CoV. Int J Infect Dis 2020;94:119-124

7 Cheng Y, Wong R, Soo YO, et al. Use of convalescent plasma therapy in SARS patients in Hong Kong. Eur J Clin Microbiol Infect Dis 2005;24(01):44-46

8 Arabi YM, Hajeer AH, Luke T, et al. Feasibility of using convalescent plasma immunotherapy for MERS-CoV infection, Saudi Arabia. Emerg Infect Dis 2016;22(09):1554-1561

9 Chen L, Xiong J, Bao L, Shi Y. Convalescentplasma as a potential therapy for COVID-19. Lancet Infect Dis 2020;20(04):398-400

10 Tiberghien P, de Lamballerie X, Morel P, Gallian P, Lacombe K, Yazdanpanah Y. Collecting and evaluating convalescent plasma for COVID-19 treatment: why and how? Vox Sang 2020 (e-pub ahead of print) . Doi: 10.1111/vox.12926

11 Wong HK, Lee CK. Pivotal role of convalescent plasma in managing emerging infectious diseases. Vox Sang 2020 (e-pub ahead of print). Doi: 10.1111/vox.12927

12 Roback JD, Guarner J. Convalescent plasma to treat COVID-19: possibilities and challenges. JAMA 2020 (e-pub ahead of print) . Doi: $10.1001 /$ jama.2020.4940

13 Bloch EM, Shoham S, Casadevall A, et al. Deployment of convalescent plasma for the prevention and treatment of COVID-19. J Clin Invest 2020;130(06):2757-2765

14 Duan K, Liu B, Li C, et al. Effectiveness of convalescent plasma therapy in severe COVID-19 patients. Proc Natl Acad Sci U S A 2020;117(17):9490-9496

15 World Health Organization. Clinical management of severe acute respiratory infection when Novel coronavirus (nCoV) infection is suspected: Interim guidance. Available at: https://www.who.int/ publications-detail/clinical-management-of-covid-19. Accessed June 2, 2020

16 Shen C, Wang Z, Zhao F, et al. Treatment of 5 critically ill patients with COVID-19 with convalescent plasma. JAMA 2020;323(16): 1582-1589
17 Rajendran K, Krishnasamy N, Rangarajan J, Rathinam J, Natarajan M, Ramachandran A. Convalescent plasma transfusion for the treatment of COVID-19: systematic review. J Med Virol 2020 (epub ahead of print). Doi: 10.1002/jmv.25961

18 Perotti C, Del Fante C, Baldanti F, et al. Plasma from donors recovered from new Corona virus 2019 as therapy for critical patients with COVID-19 (COVID-19 Plasma study). A multicentre study protocol. Intern Emerg Med 2020 [epub ahead of print] doi: 10.1007/s11739-020-02384-2

19 Franchini M, Marano G, Velati C, Pati I, Pupella S, Liumbruno G. Operational protocol for donation of anti-COVID-19 convalescent plasma in Italy. Vox Sang 2020 (e-pub ahead of print) . Doi: 10.1111/vox. 12940

20 Franchini M, Farrugia A, Velati C, et al. The impact of the SARSCoV-2 outbreak on the safety and availability of blood transfusions in Italy. Vox Sang 2020 (e-pub ahead of print) . Doi: $10.1111 /$ vox. 12928

21 Li G, Fan Y, Lai Y, et al. Coronavirus infections and immune responses. J Med Virol 2020;92(04):424-432

22 Lin Q, Zhu L, Ni Z, Meng H, You L. Duration of serum neutralizing antibodies for SARS-CoV-2: lessons from SARS-CoV infection. J Microbiol Immunol Infect 2020 (e-pub ahead of print) . Doi: 10.1016/j.jmii.2020.03.015

23 Zhou G, Zhao Q. Perspectives on therapeutic neutralizing antibodies against the Novel Coronavirus SARS-CoV-2. Int J Biol Sci 2020;16(10):1718-1723

24 Wang F, Hou H, Luo Y, et al. The laboratory tests and host immunity of COVID-19 patients with different severity of illness. JCI Insight 2020;5(10):137799

25 Lindholm PF, Ramsey G, Kwaan AC. Passive immunity for COVID 19-a commentary on therapeutic aspects including convalescent plasma. Semin Thromb Hemost 2020 (e-pub ahead of print) . Doi: $10.1055 / \mathrm{s}-0040-1712157$ 